\title{
Validación y aplicación de un instrumento para medir el conocimiento sobre radioprotección en alumnos de posgrado
}

Validation and application of an instrument to measure radioprotection knowledge of postgraduate students.

\author{
Anne Marie Kusch 1,a;,,b, Vilma Ruiz García ${ }^{2, a, c, d}$
}

\section{RESUMEN}

Objetivo: Medir el conocimiento general sobre las medidas de protección radiológica, beneficios y riesgos de los exámenes auxiliares en los alumnos de posgrado. Material y Métodos: Se confeccionó un cuestionario que constó de 20 preguntas, el que posteriormente fue validado por un juicio de expertos que fue evaluado a través $\mathrm{V}$ de Aiken $(0,89)$. Se realizó una prueba "test y retest" en 25 alumnos de postgrado, obteniendo un coeficiente de Pearson de 0,89 y la fiabilidad del instrumento fue evaluada a través de alfa de Cronbach con un valor de 0,79. Se aplicaron 194 encuestas a los alumnos de postgrado de las 12 especialidades impartidas por la FE-UPCH, en base al sistema vigesimal establecido por el Ministerio de Educación de Perú, se consideraron aprobados a quienes obtuvieron una calificación final mayor o igual a 11. Resultados: Se observó una mayor tendencia de conocimiento en el sexo femenino, en la modalidad de ingreso vía vacante ordinaria semi-presencial y en la segunda especialidad de Radiología Bucal y Máxilofacial Semi-presencial. Conclusiones: El conocimiento general de los alumnos de postgrado de la FE-UPCH con respecto a medidas de protección radiológica, beneficios y riesgos de los exámenes auxiliares es bueno, más de la mitad de ellos aprobaron el cuestionario aplicado.

PALABRAS CLAVE: Estudios de validación, conocimiento, protección radiológica.

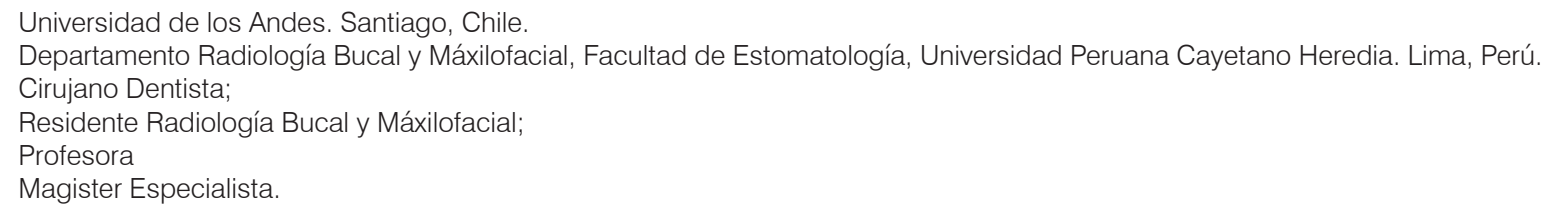




\section{SUMMARY}

Objective: Messure the knowledge of postgraduates studens of the Universidad Peruana Cayetano Heredia (UPCH) about radiation protection, benefits and risks of auxiliary imaging exams in the period November (2017) to February (2018). Material and Methods: a 20 questions questionnaire was prepared, subsequently was validated by an expert judgement, this was evaluated through V de Aiken (0.89). A test and retest was carried out in 25 postgraduate students, obtaining a Pearson coefficient of 0.89 and a realiability of 0.79 through Cronbach's alpha. 194 surveys were applied to the students of the 12 different programs of the FE-UPCH, based on the vigesimal system established by the Ministry of Education, those who obtained a final grade greater than or equal to 11 were considered approved. Results: It was observed a greater tendency of knowledge in female sex, in the modality of income via ordinary semi-presential modality vacancy and in the second specialty of oral and maxillofacial semi-face radiology. Conclusions: general knowledge of the postgraduate students of the UPCH regarding radiological protection measures, benefits and risks of the auxiliary exams is good; more than half of them approved the applied questionnaire.

\section{KEYWORDS: Validation studies, knowledge, radiation protection.}

\section{INTRODUCCIÓN}

Los exámenes radiológicos y de medicina nuclear son considerados actualmente como grandes y esenciales herramientas diagnósticas y por ende, forman parte de la base de numerosas decisiones terapéuticas. Estas imágenes diagnósticas dependen fundamentalmente de radiación del tipo ionizante, siendo los rayos $\mathrm{X}$ los más ampliamente difundidos por su gran capacidad de penetración en la materia $(1,2)$. Casi la mitad de la exposición a la radiación que una persona recibe a lo largo de su vida es debida a procedimientos relacionados con su salud (2).

Con fines médicos se han venido utilizando métodos de radiación ionizante por más de un siglo. Los beneficios se encuentran indudablemente presentes y éstos están por sobre los posibles riesgos. No se puede negar que los avances en la tecnología y su contribución a la medicina han permitido salvar la vida de numerosos pacientes y han revolucionado la práctica de la medicina (3).

La radiación ionizante es un tema muy amplio, complicado y normalmente malentendido. Su exposición sigue estando asociada a estadios agudos y crónicos de enfermedades $(4,5)$. Conforme las tecnologías van evolucionando y mejorando la cantidad de radiación a la que se exponen los pacientes, así como el personal auxiliar, ha ido disminuyendo la dosis de radiación. Cada vez los esfuerzos son mayores hacia conseguir una mejor imagen utilizando una menor dosis de radiación. Es por esto, que los efectos biológicos resultantes de grandes exposiciones a radiaciones ya no son motivo de estudios, sino que ahora se busca estudiar los efectos a largo plazo de los niveles bajos de radiación (6).

Existe en la población un sentimiento de temor frente a la realización de estos exámenes, esto es causado en gran medida por los medios de comunicación, que entregan una información errónea, exagerando situaciones y también minimizando otras. El lenguaje técnico empleado por el profesional juega un rol esencial, ya que puede llevar a la falta de comprensión de lo que se está comunicando y por ende a confusiones por parte del paciente $(7,8)$.

Es responsabilidad de todos los profesionales relacionados a la salud entregar al paciente toda la información existente, beneficios y riesgos antes de que este se someta a un examen imagenológico; ayudándolo de esta manera a tomar una decisión informada con respecto al procedimiento a realizar $(9,10)$. Para cumplir esto, todo profesional que indique este tipo de exámenes auxiliares debe estar correctamente instruido. Sin embargo, hay diversos estudios que exponen que existen muchos profesionales que podrían no estar tan informados como el paciente espera $(1,11)$. Muchos tratantes tienden a subestimar las dosis de radiación emitidas por los equipos médicos y muchas veces, inclusive, no tienen conciencia sobre qué exámenes imagenológicos son fuentes de emisión de radiación $(12,13)$.

El objetivo del presente trabajo fue evaluar los conocimientos que poseen los alumnos de posgrado de 
la Facultad de Estomatología de la Universidad Peruana Cayetano Heredia (FE- UPCH) sobre medidas de protección radiológicas, riesgos y beneficios que la exposición a radiación ionizante representa a los pacientes mediante un cuestionario elaborado por las autoras, el que fue previamente validado mediante un juicio de expertos.

\section{MATERIAL Y METODOS}

La investigación fue aprobada por el Comité Institucional de Ética de la Universidad Peruana Cayetano Heredia con documento número 645-21-17, brindando la categoría de exento.

El estudio fue observacional, descriptivo y transversal, realizado entre los años 2017 y 2018. Luego de la elaboración y posterior validación del cuestionario, se administró a todos los alumnos de posgrado con matrícula vigente y asistencia regular.

El cuestionario constó de dos partes: la primera de preguntas de conocimientos sobre protección radiológica y otra tuvo preguntas de conocimientos de los beneficios y riesgos a la exposición de radiación ionizante. Este fue aplicado a los alumnos previa coordinación con los encargados de cada área; sin haber recibido formación antes sobre el tema a evaluar en clases ni seminarios previamente a la aplicación. Los datos obtenidos fueron analizados en Excel (Microsoft ${ }^{\circledR}$ 2011) e interpretados en base a análisis descriptivos. Como no se realizaron procedimientos médicos ni se recolectó data que pudiese vulnerar la intimidad de los participantes, bastó con la entrega de una hoja informativa y no se precisó el uso de un consentimiento informado.

\section{RESULTADOS}

Para la validación del contenido, mediante el juicio de expertos, se empleó el método estadístico $\mathrm{V}$ de Aiken, obteniendo un valor de 0,78 .

Para la reproducibilidad (test y retest) se realizó un estudio piloto con una muestra formada por 25 alumnos de 3 programas de postgrado de la FE-UPCH, evaluándose a través del coeficiente de Pearson el que tuvo un valor de 0,89 .
La fiabilidad del instrumento fue medida a través del método estadístico Alfa de Cronbach, obteniéndose un valor de 0,79 .

Basado en el sistema de evaluación peruano, cada encuesta fue evaluada con una calificación que va entre 0 a 20. Se consideraron aprobados a todos aquellos que obtuvieran un puntaje mayor o igual a 11, según sistema escala vigesimal de evaluación del Ministerio de Educación (14).

Un total de 194 alumnos de posgrado de la FE-UPCH fueron encuestados; de ellos 115 (59,28\%) corresponden a al sexo femenino (M) y $79(40,72 \%)$ al sexo masculino $(\mathrm{H})$, pertenecientes a las 12 especialidades que imparte la Facultad de Estomatología, incluyendo al programa CODIRO (Programa de Residentado Nacional Odontológico) según cada especialidad (tabla 1). Siendo el programa de Ortodoncia el que posee mayor cantidad de alumnos matriculados y Medicina y Patología Oral el que menor cantidad de alumnos matriculados posee; así como Rehabilitación Oral en su programa CODIRO.

Al evaluar el conocimiento de los alumnos con respecto al sexo se observó una tendencia de mayor conocimiento en $\mathrm{M}$ que en $\mathrm{H}$ matriculados (tabla 2).

Se observó que existe una tendencia de mayor aprobación en aquellos alumnos de la especialidad de Radiología Oral y Máxilofacial modalidad semipresencial con 11 alumnos aprobados (91,67\%), y por otro lado la especialidad de Periodoncia e Implantología fue quién presentó una tendencia de mayor desaprobación, aprobando sólo 5 alumnos $(55,56 \%)$ (tabla 3$)$.

Se evidenció que la pregunta que tuvo mayor acierto fue la número 20 siendo 188 (96,91\%) alumnos que la respondieron de manera correcta, la pregunta con menor aciertos fue la número 9 siendo contestada de manera correcta sólo por 46 (23,71\%) alumnos ( tabla 4). 
Tabla 1. Distribución de alumnos de posgrado según programa de segunda especialidad

\begin{tabular}{lcc}
\multicolumn{1}{c}{ Programa de posgrado } & \multicolumn{2}{c}{ Total } \\
& n & $\%$ \\
\hline Endodoncia & 15 & 7,73 \\
Endodoncia CODIRO & 4 & 2,06 \\
Cirugía Oral y Máxilofacial & 16 & 8,25 \\
Cirugía Oral y Máxilofacial CODIRO & 1 & 0,52 \\
Periodoncia e Implantología & 9 & 4,64 \\
Implantología Oral Integral & 6 & 3,09 \\
Ortodoncia y Ortopedia Maxilar & 45 & 23,20 \\
Odontopediatría & 15 & 7,73 \\
ROMF* Presencial & 9 & 4,64 \\
ROMF Semi-presencial & 12 & 6,19 \\
ROMF CODIRO & 6 & 3,09 \\
EPE** & 4 & 2,06 \\
EPE CODIRO & 2 & 1,03 \\
Medicina y Patología Estomatológica & 2 & 1,03 \\
Rehabilitación Oral & 23 & 11,86 \\
Rehabilitación Oral CODIRO & 1 & 0,52 \\
Salud Pública CODIRO & 9 & 7,73 \\
Odontología Restauradora y Estética & & 4,64 \\
\hline
\end{tabular}

*ROMF: Radiología Oral y Máxilofacial.

**EPE: Estomatología de Pacientes Especiales.

Tabla 2. Conocimiento según sexo

\begin{tabular}{ccccc}
\hline \multicolumn{4}{c}{ Sexo } & \multicolumn{3}{c}{ Conoce } \\
& $\mathrm{n}$ & $\%$ & $\mathrm{n}$ & $\%$ \\
\hline Masculino & 59 & 30,41 & 20 & 10,31 \\
Femenino & 82 & 42,27 & 33 & 17,01 \\
\hline
\end{tabular}


Tabla 3. Conocimiento de acuerdo a programa de segunda especialidad

\begin{tabular}{|c|c|c|c|c|}
\hline \multirow{2}{*}{ Programa } & \multicolumn{2}{|c|}{ Conoce } & \multicolumn{2}{|c|}{ No conoce } \\
\hline & $\mathbf{n}$ & $\%$ & n & $\%$ \\
\hline Endodoncia & 9 & 60 & 6 & 40 \\
\hline Endodoncia CODIRO & 4 & 100 & 0 & - \\
\hline Cirugía Oral y Máxilofacial & 11 & 68,75 & 5 & 31,25 \\
\hline Cirugía Oral y Máxilofacial CODIRO & 1 & 100 & 0 & - \\
\hline Periodoncia e Implantología & 5 & 55,56 & 4 & 44,44 \\
\hline Implantología Oral Integral & 4 & 66,67 & 2 & 33,33 \\
\hline Ortodoncia y Ortopedia Maxilar & 34 & 75,56 & 11 & 24,44 \\
\hline Odontopediatría & 9 & 60 & 6 & 40 \\
\hline ROMF* Presencial & 7 & 77,78 & 2 & 22,22 \\
\hline ROMF Semi-presencial & 11 & 91,67 & 1 & 8,33 \\
\hline ROMF CODIRO & 5 & 83,33 & 1 & 16,67 \\
\hline $\mathrm{EPE}^{* *}$ & 4 & 100 & 0 & - \\
\hline EPE CODIRO & 2 & 100 & 0 & - \\
\hline Medicina y Patología Estomatológica & 2 & 100 & 0 & - \\
\hline Rehabilitación Oral & 15 & 65,22 & 8 & 34,78 \\
\hline Rehabilitación Oral CODIRO & 1 & 100 & 0 & - \\
\hline Salud Pública CODIRO & 11 & 73,33 & 4 & 26,67 \\
\hline Odontología Restauradora y Estética & 8 & 88,89 & 1 & 11,11 \\
\hline
\end{tabular}

*ROMF: Radiología Oral y Máxilofacial.

**EPE: Estomatología de Pacientes Especiales. 
Tabla 4. Porcentaje de respuestas correctas según pregunta planteada.

\begin{tabular}{|c|c|c|c|}
\hline & & Correctamente & respondidas \\
\hline & Pregunta & n & $\%$ \\
\hline 1) & ¿Con qué Kv (kilovoltaje) funciona un equipo radiográfico intraoral? & 60 & 30,93 \\
\hline 2) & ¿Qué mA (miliamperaje) utiliza un equipo radiográfico intraoral? & 62 & 31,96 \\
\hline 3) & ¿Cree Ud. que se pueden tomar radiografías a niños de cualquier edad? & 101 & 52,06 \\
\hline 4) & $\begin{array}{l}\text { ¿Sigue Ud. en su práctica diaria, en cuanto a radiación, el principio de } A L A R A \\
\text { (lo más bajo como sea razonablemente posible)? }\end{array}$ & 150 & 77,32 \\
\hline 5) & $\begin{array}{l}\text { ¿Cree Ud. que las radiaciones controladas representan algún riesgo para nuestra } \\
\text { salud? Considere que ellas conviven con nosotros en hospitales, industrias, gas- } \\
\text { es, diagnóstico y tratamiento de enfermedades? }\end{array}$ & 84 & 43,30 \\
\hline 6) & $\begin{array}{l}\text { Al comparar una radiografía de cráneo con una radiografía de uso odontológico, } \\
\text { ¿Cuál cree Ud. que tiene mayor dosis de radiación? }\end{array}$ & 141 & 72,68 \\
\hline 7) & Según su opinión, ¿Qué examen tendrá mayor dosis de radiación? & 59 & 30,41 \\
\hline 8) & ¿El daño de los rayos $\mathrm{X}$ en tejidos corporales se debe a...? & 111 & 57,22 \\
\hline 9) & ¿Pueden realizarse tomas de radiografías periapicales en mujeres embarazadas? & 46 & 23,71 \\
\hline 10) & ¿Tienen las radiografías efectos secundarios en los pacientes? & 122 & 62,89 \\
\hline 11) & $\begin{array}{l}\text { Con respecto a la dosis de radiación: Una dosis de radiación baja pero que se } \\
\text { aplica durante un periodo prolongado ¿tendría riesgo para el paciente? }\end{array}$ & 153 & 78,87 \\
\hline 12) & $\begin{array}{l}\text { Los pacientes que han sido expuestos con anterioridad (por motivos médicos) } \\
\text { a radiación ionizante por un largo periodo de tiempo ¿Tienen mayor riesgo de } \\
\text { sufrir cáncer aunque reciban dosis bajas de radiación? }\end{array}$ & 115 & 59,28 \\
\hline 13) & ¿Es la sensibilidad a la radiación ionizante directamente proporcional a la edad? & 87 & 44,85 \\
\hline 14) & $\begin{array}{l}\text { ¿Cree Ud. que dosis altas de radiación podrían ser beneficiosas para un paciente } \\
\text { enfermo de cáncer? }\end{array}$ & 84 & 43,30 \\
\hline 15) & $\begin{array}{l}\text { ¿Sabía Ud. que en la actualidad existen otras aplicaciones de la radiación ion- } \\
\text { izante como lo es su aplicación para esterilizar pabellones quirúrgicos, entre } \\
\text { otros? }\end{array}$ & 95 & 48,97 \\
\hline 16) & $\begin{array}{l}\text { ¿Cree Ud. que las radiografías constituyen una necesidad para poder dar un } \\
\text { diagnóstico certero? }\end{array}$ & 157 & 80,93 \\
\hline 17) & $\begin{array}{l}\text { ¿Qué órganos del cuerpo requieren de protección para una toma radiográfica } \\
\text { dental? }\end{array}$ & 145 & 74,74 \\
\hline 18) & ¿Usa el delantal de plomo en sus pacientes durante el examen radiológico? & 181 & 93,30 \\
\hline 19) & ¿Usa collar tiroideo en sus pacientes durante el examen radiológico? & 155 & 79,90 \\
\hline 20) & $\begin{array}{l}\text { ¿Cree Ud. que es de utilidad tener plomadas las paredes, que rodean a un equipo } \\
\text { que emite radiación ionizante? }\end{array}$ & 188 & 96,91 \\
\hline
\end{tabular}




\section{DISCUSIÓN}

Al ser una investigación de tipo descriptivo, permite establecer tendencias pero no una conclusión absoluta; esto no es menos enriquecedor ya que nos brinda datos con respecto a la frecuencia de aparición del fenómeno que queremos evaluar, es la base de otros estudios como los de factores asociados a la aparición del problema y por último, informa no sólo a los sujetos del estudio sino también a la comunidad acerca de la situación en la que se encuentran con respecto al problema; permitiendo establecer un punto de partida para la planificación en los sistemas de salud (15).

Es deficiente la aprobación que se obtuvo con respecto a la información de Kv y mA con que operan los equipos radiológicos en Perú, sólo un 30,93\% respondió de manera correcta sobre el Kv y un 31,96\% sobre el mA. Esto coincide con el poco conocimiento de este ítem en otros países como India, donde Pal et al., al encuestar a 156 dentistas vieron que un $82.3 \%$ no sabía el valor de $\mathrm{Kv}$ en que operaban los equipos imagenológicos dentales (16). Esto puede explicarse por el hecho de que los equipos periapicales actualmente vienen con los valores de $\mathrm{Kv}$ y $\mathrm{mA}$ ya programados y fijos, evitando así que el operador manipule estos factores. Esto facilita el procedimiento de la toma, pero genera desconocimiento por parte del operador en cuanto a términos de radioprotección para los pacientes.

Con respecto a realizar la práctica clínica diaria bajo el principio ALARA (As Low As Reasonably Achievable; tan bajo como sea razonablemente posible), un $77,32 \%$ de los encuestados afirmó seguirlo. Aravind et al.,encontraron una cifra superior, un 84,3\% (17), similar al 85,3\% observado por Berg et al., (18); en cambio, Enabulele e Itimi señalan que en su encuesta sólo un $18.6 \%$ sabe qué significaba dicho término (19). Esta última situación podría explicarse por un tema cultural. No hasta hace mucho tiempo se consideraba necesaria la inclusión de temas de bioseguridad y radioprotección en la educación y formación de los estudiantes del área de la salud (20).

Enabulele e Itimi en el año 2015 encuestaron a 85 estudiantes de la carrera de odontología, la mitad de ellos respondieron que los daños que provocaban los rayos $\mathrm{X}$ se debían a la acción de radicales libres, es decir, a efectos indirectos (19). En la presente investigación la mayoría de los alumnos $(57,22 \%)$ contestó que el daño que se produce en los tejidos corporales se debe tanto a efectos directos como indirectos; cifras muy superiores a las observadas por Aravind et al. donde un $88 \%$ ni siquiera sabían sobre los efectos biológicos de la radiación ionizante (21); similar a lo observado por Razi et al.,donde evidenciaron que el $70 \%$ de los dentistas generales no posee conocimientos sobre los efectos biológicos de la radiación ni cómo se producen (22). Esta tendencia se puede explicar por el hecho de que dentro de la malla de los programas de posgrado que ofrece la institución donde se realizó la investigación; se imparte a los ingresantes cursos intensivos de actualización, en donde se tocan tópicos selectos puntuales.

Un $23,71 \%$ considera que se puede tomar radiografías dentales en cualquier periodo de gestación; cifra superior a la obtenida por Pal et al., quienes vieron sólo un $16.7 \%$ (16), pero similar a la observada por Aravind et al., con un 22\% (21). La mayoría de los profesionales encuestados por Chaudhry et al., preferían no tomar ningún tipo de radiografía dental durante toda la gestación (23). Este resultado podría verse explicado por el hecho de que existe el concepto preconcebido de que el feto en formación es un ser sensible al medio externo y que podría sufrir daños irreversibles con la radiación emitida por los equipos radiológicos dentales; por el hecho de que el contenido en los cursos de embriología no siempre contemplan la potencialidad real de la teratogenicidad de los rayos $\mathrm{X}$ y también como posible causa de que muchos estudiantes e inclusive profesionales no distinguen bien las diferentes técnicas radiográficas ni sus dosis de radiación.

El 44,85\% de los encuestados contestó de manera correcta que la sensibilidad a la radiación es proporcional a la edad. Cifra mayor a la observada por Aravind et al.,con un $22 \%(21)$.

El $74,74 \%$ de los encuestados contestó de manera correcta que es el órgano de la tiroides la que requiere protección durante la toma de una radiografía dental. Similar a lo observado por Chaudhry et al., en el año 2016 , con un $64.8 \%$ (23); situación que también puede verse explicada por la realización de cursos intensivos antes del inicio de cada programa de especialidad.

En el presente estudio la mayoría de los estudiantes de postgrado utiliza el delantal plomado y collar tiroi- 
deo para la realización de la técnica radiográfica con un $93,30 \%$ y $79,90 \%$ respectivamente, similar a lo observado por Mojiri y Moghimbeigi con un 78,9\% y $83,1 \%$ respectivamente (24); esta información coincide con lo observado por An et al., los que encuestaron a 207 dentistas de Korea, todos respondieron que utilizaban ambas barreras de protección (25). Esto se opone a lo observado por Pal et al., donde la mayoría de sus encuestados respondió que no utilizan ni delantal plomado ni collar tiroideo (16), similar a lo observado por Noohi con un 84,3\% (26); así como Aravind et al., quiénes encuestaron a 300 dentistas el año 2016 , observando que un $90.3 \%$ no las utiliza (21). Chaudhry et al., observaron cifras aún mayores, un $98 \%$ de los profesionales encuestados no utiliza barreras de protección en sus pacientes (23).

Nuevamente esta situación podría explicarse por un tema cultural en el orden de los nuevos paradigmas de enseñanza en las ciencias de la salud. Los tópicos de bioseguridad así como los de radioprotección son recientes en la currícula, no habiendo sido incluidos de manera universal en todas las instituciones académicas superiores.

Un 96,91\% considera útil que las paredes se encuentren plomadas para proteger a operadores coincidiendo con lo observado por Enabulele e Itimi con cifras elevadas de sus encuestados (92,5\%) (19).

Con respecto a las otras preguntas, no existe información disponible en la literatura para poder contrastar. Generando una situación propicia para iniciar una línea de investigación, ya que, al tener un instrumento creado y validado, este se puede aplicar en diferentes latitudes para luego contrastar resultados; ya que una vez sometido al estricto proceso de validación éste sólo debe adaptarse al entorno o idioma para su aplicación.

El estudio no estuvo exento de dificultades, como lo fue el aplicar las encuestas a aquellos alumnos matriculados que se encontraban rotando en centros hospitalarios fuera de la universidad, o a aquellos que no asistieron a la clase el día que estaba programada la aplicación de las mismas.

\section{CONCLUSIONES}

El conocimiento general de los alumnos de postgrado de la FE-UPCH con respecto a medidas de protección radiológica, beneficios y riesgos de los exámenes auxiliares es bueno.

Se observó que el sexo femenino tiene un mayor conocimiento con respecto al sexo masculino.

El programa con mayor conocimiento corresponde al de ROMF semi-presencial y el de menor al de Periodoncia e Implantología.

Existe mayor conocimiento en aquellos alumnos que ingresaron vía ordinaria semi-presencial y un menor conocimiento en aquellos que ingresaron vía ordinaria presencial.

Es deber de las instituciones, ya sean establecimientos educativos o laborales, asegurarse de reforzar las medidas de bioseguridad generales y específicas (por ejemplo, de radioprotección), según el área, actualizar a su personal de manera constante y además velar que cuenten con los implementos necesarios para ello.

\section{Correspondencia:}

Anne Marie Betty Kusch Noelke

Correo electrónico: anne.kusch@upch.pe

\section{REFERENCIAS BIBLIOGRÁFICAS}

1. Fazel R, Krumholz HM, Wang Y, et al. Exposure to low-dose ionizing radiation from medical imaging procedures. N Engl J Med. 2009;9:849-57.

2. Hall EJ, Giaccia A. Radiobiology for the Radiologist. 7th ed. Philadelphia: Wolters Kluwer Health/Lippincott Williams \& Wilkins; 2012.

3. Amis ES, Butler PF, Applegate KE, et al. American College of Radiology White Paper on Radiation Dose in Medicine. J Am Coll Radiol. 2007;4(5):272-84.

4. Christodouleas JP, Forrest RD, Ainsley CG, Tochner Z, Hahn SM, Glatstein E. Short-term and long-term health risks of nuclear-power-plant accidents. N Engl J Med. 2011;24:2334-41.

5. Linet MS, Slovis TL, Miller DL, et al. Cancer risks associated with external radiation from diagnostic imaging procedures. CA Cancer J Clin. 2012;62(2):75-100.

6. Hendee WR. Real and perceived risks of medical radiation exposure. West J Med. 1983;138 (3):380-6.

7. Kanda R, Tsuji S, Yonehara H. Perceived risk of nu- 
clear power and another riks during the last 25 years in Japan. Health Phys. 2012;104(4):384-90.

8. Slovic P. Percepcion of risk from radiation. Radiat Prot Dosimetry. 1996;68(3-4):165-80.

9. Lee CI, Flaster HV, Haims AH, Monico EP, Forman HP. Diagnostic CT Scans: Institutional Informed Consent Guidelines and Practices at Academic Medical Centers. Am J Roentgenol. 2006;187(2):287-7.

10. Ludwig RL, Turner LW. Effective patient education in medical imaging: public perceptions of radiation exposure risk. J Allied Health. 2002;31(3):159-64.

11. Nickoloff EL, Alderson PO. Radiation exposures to patients from CT: reality, public perception, and policy. Am J Roentgenol. 2001;177(2):285-7.

12. Arslanoglu A, Bilgin S, Kubali Z, Ceyhan MN, İlhan MN, Maral I. Doctors' and intern doctors' knowledge about patients' ionizing radiation exposure doses during common radiological examinations. Diagn Interv Radiol. 2007;13(2):53-7.

13. Sjoberg L, Drottz-Sjoberg B-M. Knowledge and risk perception among nuclear power plant employees. Risk Anal. 1991;11(4):607-18.

14. Ministerio de Educación. Diseño Curricular Nacional. Lima: Ministerio de Educación ;2005.

15. Villavicencio E. La importancia de los estudios descriptivos. Rev Evid Odontol Clin. 2016;2(1):6-7.

16. Pal S, Bhattacharya PT, Sinha R. Radiation protection in dentistry - Do we practice what we learn? J Adv Clin Res Insights. 2015;2:155-9.

17. Aravind BS, Joy ET, Kiran MS, Sherubin JE, Sajesh S, Manchil PRD. Attitude and awareness of general dental practitioners toward radiation hazards and safety. J Pharm Bioallied Sci. 2016;8(1):58-8.
18. Berg B, Gertsch A, Zeilhofer H, et al. Digitale Volumentomographie und Strahlenbelastung. Swiss Dent J. 2014;124(4):419-26.

19. Enabulele J, Igbinedion BO. An assessment of dental students'knowledge of radiation protection and practice. J Dent Educ. 2013; 3(2): 54-9.

20. Enabulele J, Itimi E. Endodontic radiology, practice, and knowledge of radiation biology, hazard, and protection among clinical dental students and interns. Saudi Endod J. 2015;5(3):171-6.

21. Aravind BS, Joy ET, Kiran MS, Sherubin JE, Sajesh $\mathrm{S}$, Manchil PRD. Attitude and awareness of general dental practitioners toward radiation hazards and safety. J Pharm Bioallied Sci. 2016;8(Suppl 1):S53.

22. Razi T, Bazvand L, Ghojazadeh M. Diagnostic dental radiation risk during pregnancy: Awareness among general dentists in Tabriz. J Dent Res Dent Clin Dent Prospects. 2011;5:67-70.

23. Chaudhry M, Jayaprakash K, Shivalingesh K, et al. Oral Radiology Safety Standards Adopted by the General Dentists Practicing in National Capital Region (NCR). J Clin Diagn Res. 2016; 10(1): ZC42-ZC45. doi: 10.7860/JCDR/2016/14591.7088

24. Mojiri M, Moghimbeigi A. Awareness and attitude of radiographers towards radiation protection. J Paramed Sci. 2011;2(4):2-5.

25. An S-Y,Lee K-M, Lee J-S. Korean dentists'perceptions and attitudes regarding radiation safety and protection. Dentomaxillofac Radiol. 2017;47(3):20170228. doi: 10.1259/dmfr.20170228

26. Noohi J. Evaluation of Personnel protection among radiographers in diagnostics radiology centers in Kerman (Iran). Iran: Environmental Health Conference; 2009.

Recibido: 23-08-2018

Aceptado: 09-11-2018 